\title{
Molten area detection in laser cladding based on vision detection
}

\author{
YONG Yaowei ${ }^{1,2, a}$, FU Wei ${ }^{3,4, b}$, CHEN Dianbin ${ }^{1, c}$,DENG Qilin ${ }^{1, d, *}$
}

\author{
1. State Key Laboratory of Mechanical System and Vibration, Shanghai Jiao Tong University , \\ Shanghai, 200240, PR China
}

2. School of Mechanical Engineering, Ningxia University, Yinchuan Ningxia, 750021, PR China

3. State Key Laboratory of Advanced Welding and Joining, Harbin Institute of Technology, Harbin, 150001, PR China

4. BAOSTEEL Industry Technological Service Co. Ltd, Shanghai, 201900, PR China

ayywnxu@163.com; ' fw9106@163.com; ' chendanbingsjtu@163.com; dengqilin@sjtu.edu.cn

Corresponding Author: Dengqilin@sjtu.edu.cn

Keywords: laser cladding; molten pool area; vision detection

\begin{abstract}
Laser cladding has been widely introduced into industries for improving or changing the behavior of material to against corrosion, wear and so on. In the process, the molten pool, which is related to powder mass and others, is formed to melt the introduced powder onto the substrate. Thus, the area and stability of molten pool will affect not only productivity but also the final product quality. In order to obtain good quality with excellent stability molten pool in cladding process, we present a low-cost COMS vision detection system to surveille the process, which was programmed by LabVIEW. The different powders were preplaced onto the substrate with different thickness or feed with different mass flow rate, the cladding results were shown that the vision detection is very efficient and accuracy. The results show that the molten pool area increases with the thicker powder, while the dilution will decrease.
\end{abstract}

\section{Introduction}

Laser cladding is an important surface modification process that use a high power laser beam to melt the coating material and thinner layer of substrate to form a new surface with low dilution and metallurgical bond with substrate [1], and therefore it has superb combination mechanical characteristics such as good corrosion and wear resistance, high hardness and etc., even more, it can be deposited to form a surface with self-lubrication. There are many parameters affecting the process and thus quality of laser cladding, such as laser power input, size of laser beam spot and laser scanning velocity, besides, the absorption of laser by substrate is also important in laser process.

During laser cladding, the key process is that the coating materials are melted and sequent consolidated onto the substrate. Based on this understanding, appropriate parameters should be chosen on the conditions that the cladding materials be melted thoroughly without deteriorated dilution of substrate so as to achieve the dense bonding between them. Thus a number of works had been carried out to study the effect of those important process parameters in laser cladding. Qian et al. [2] performed laser cladding on AISI 1020 with Colmonoy 88 with powder feed rate varied from 10.8 to $20.4 \mathrm{~g} / \mathrm{min}$ and scanning speed ranging from 350 to600 $\mathrm{mm} / \mathrm{min}$. The results concluded that the ration of width/height of cladding track and extent of dilution increase with low powder feed rate and high scanning speed, thus, the optimized powder feed rate should be $20.4 \mathrm{~g} / \mathrm{min}$ and scanning speed is $350-450 \mathrm{~mm} / \mathrm{min}$. Zhou et al. [3] studies focused on the relationship between laser cladding parameters and geometrical characteristics of each track. The experimental studies also shown that the dilution of coating is dependent on substrate type, and it increases with larger laser specific energy and substrate temperature, but the more powder feed will reduce the dilution. 
In fact, the quality of cladding coating is depending on the input parameters and working settings. Inappropriate input and setting will cause the large hear affected zone, low productivity and defects on the coating, whereas the molten pool can reflect those input and settings in laser cladding [4], thus many works had been done to observe and investigate the molten pool[5-8]. In those measures, the on-line monitoring and process system is the promising way to improve the reliability of laser cladding. Meriaudeau $[9,10]$ utilized two CCD camera to measure the surface temperature and the powder distribution and geometric dimensions, they built the linear relationship between gray pixel and temperature and announced the error is limited in 15 degrees in Celsius, the parameters such as width, length and etc. can be extracted from the CCD detection. The temperature signal was captured by photodiode and quotient pyrometer, and thus identified and qualified by Guijun Bi [4], and the molten pool size was obtained by CCD camera, ultimately, the system was built to monitor process of laser cladding.

Nevertheless, the information from the molten pool is ranged in surface temperature, irradiation intensity, pool surface area and etc. Until now, the temperature obtained from the experimentally studies are still on dispute, and have no much works on the multi-tracks of information extracted, as well, merely little attention on the substrate state. So the aims of this study are to investigate the molten pool area and quality of cladding coating surface related single and multi-track laser cladding.

\section{Experimental procedure}

\subsection{Experimental setup}

Specimens of AISI 1045 carbon steel with dimensions of $80 \mathrm{~mm} \times 40 \mathrm{~mm} \times 10 \mathrm{~mm}$ were selected as the substrates, and there had been milled onto the surface with depth of $0.5,1.0$ and $1.5 \mathrm{~mm}$. The surfaces of the samples were cleaned with steel wire brush, polished with sandpaper, dried and finally rinsed by acetone prior to laser cladding. The powder mixtures were preplaced into the milled grooves of the substrate to make sure the thickness of prefabricated power.

The powder the coating alloy were Ni55, SF60 and Fe327. The composition of alloy powder were listed in Table 1. SF60 and Fe327 were used as preplace powder. While the Ni55 was used to clad multi-track coating and given by powder feeder, and the mass flow rate were set to 3, 6, 9 and 12 $\mathrm{g} / \mathrm{min}$ under the protection of argon, the overlapping ratio was $30 \%$.

Table 1: Compositions of alloy powder

\begin{tabular}{ccccccccccc}
\hline & C & Cr & Si & W & Mo & Ni & Co & Fe & Mn & B \\
\hline Ni55 & 0.75 & 15.5 & 4 & - & 3 & Bal. & - & 14 & - & 3 \\
SF6 & - & 29 & 1.1 & 4 & 1 & 3 & Bal. & 3 & 0.5 & - \\
Fe327 & 2.5 & 10 & 2 & 5 & - & 10 & - & Bal. & - & 2.5 \\
\hline
\end{tabular}

The experiments were carried out using a $3 \mathrm{~kW}$ transverse flow continuous wave $\mathrm{CO}_{2}$ laser. The laser beam was focused using a reflective copper mirror, which will give a laser spot size on the substrate of approximately $4 * 6 \mathrm{~mm}^{2}$. Based on the previous exploring experiments, the optimized processing parameters had been determined. The laser output power was set to $1.8 \mathrm{~kW}$, the laser beam scanning velocity was $80 \mathrm{~mm} / \mathrm{min}$ in single track cladding while the power was up to $2.3 \mathrm{KW}$ and scanning velocity was set to $100 \mathrm{~mm} / \mathrm{min}$ in multi-track cladding. After laser cladding, the cross section of coating was cut, mounted and polished. They are etched by $4 \%$ nitric acid alcohol for morphology examination. The metallurgical microscope (model: MOTIC-SMZ-168) was utilized to observe the cross section and measure the geometrical size by build-in software.

The aim of this study was to observe the area of molten pool area in single and multi-track cladding. The experiments were categorized into two groups. In the first group, the thickness of powder was different, scanning SF6 and Fe327 in one track. In the second group, the multi-track laser cladding was conducted to make sure the relationship between the mass flow rate of powder and quality of coating surface. 


\subsection{Vision Detection Setup and Principle}

The vision detection system is built with a set of high speed COMS camera and LabVIEW graphic program. The molten pool state was captured by camera, whose FPS is 60, and then saved in .avi format files on driver.

The CCD camera was mounted with an angle of 40 degrees, and distanced 300mm from the focus point. To ensure the enough stiffness between the links, the system was fixed with the laser head by a steel plate of $5 \mathrm{~mm}$, and moved together, as shown in Fig 1. The direction of substrate moving is parallel to feed direction because this direction is beneficial to catch the powder.

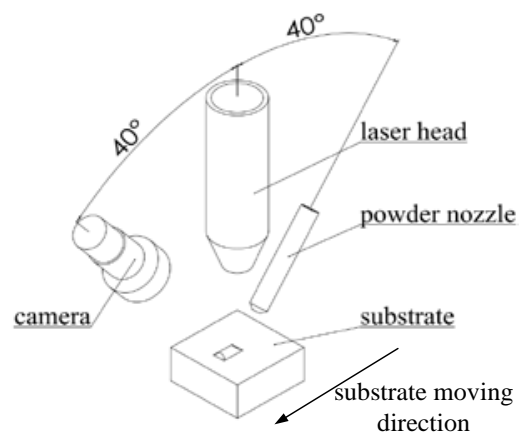

Fig. 1 Camera installation and substrate moving direction

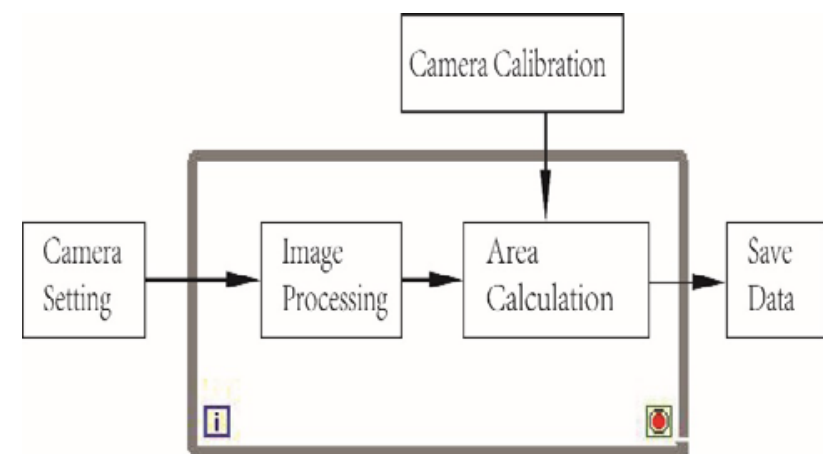

Figure 2: Diagram of Image Process

The LabVIEW software package realized the image processing, and the NI Version Development Modular was as a supplementary tool to finish process. The main image processing steps including noise filtering, thresholding, calibrating, and segmenting. The images were firstly processed with Gauss-smoothing filter shaped 3 by 3 convolution kernel to remove single bright pixels which may be caused by hot particles. Next, the image was converted into a binary image to threshold, whose value is decided according to the melt pool boundary by method of gray threshold segmentation and was 185 in future works. The third step involved the appropriate gray-level morphological close operation, which can smooth the extractive boundary. Calibrating was used to obtain the real molten pool size because the camera was mounted at a 40 angle, it not only corrects the error come from the distance of setup but also adjust the image distortion. The final process focused on the extraction of melt pool area. The diagram of image process was expressed on Fig.2. The captured image and processed image were shown in Fig 3.

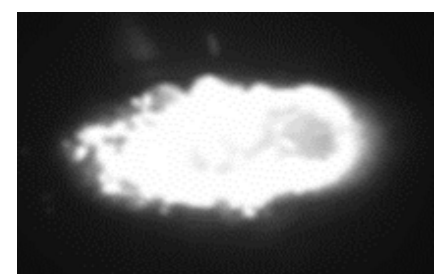

a) Original

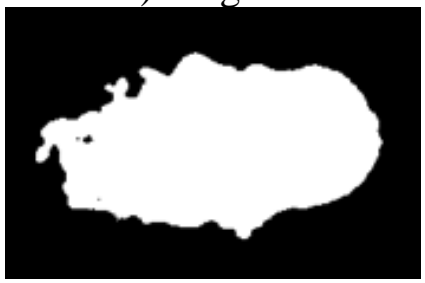

c) Segmentation with threshold

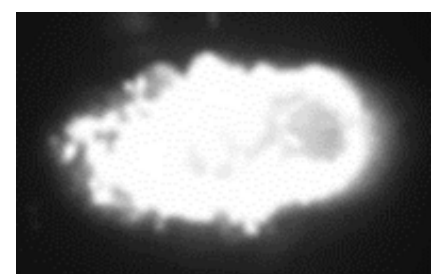

b) filter



d) final result

Figure 3: Captured image by CCD and processed image 


\section{Results and discussion}

\subsection{Different powder thickness}

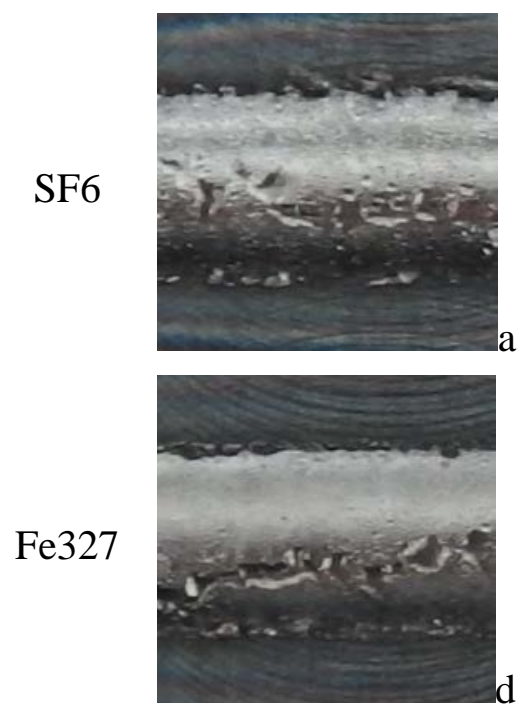

$0.5 \mathrm{~mm}$
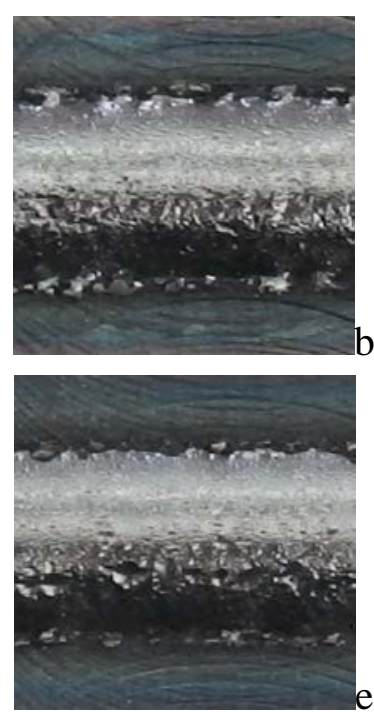

$1.0 \mathrm{~mm}$
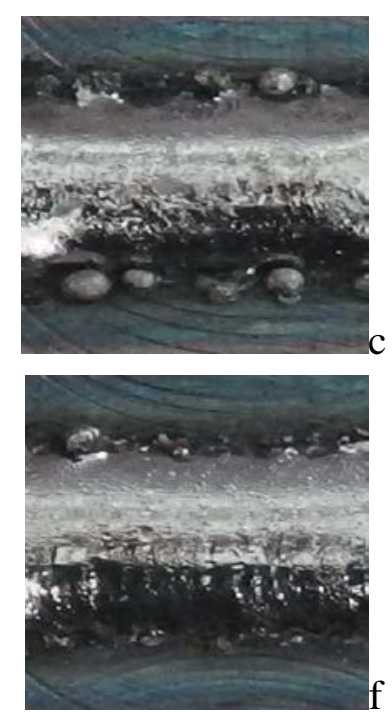

$1.5 \mathrm{~mm}$

Fig.4 Cladding track with different powder thickness

Fig. 4 show the single track of SF6 and Fe327 powder with scanning speed of $80 \mathrm{~mm} / \mathrm{min}, 1800 \mathrm{~W}$ in power and preplaced powder thickness of $0.5 \mathrm{~mm}, 1 \mathrm{~mm}$ and $1.5 \mathrm{~mm}$.

Fig. 4 a)-c) reveal that coating surface roughness, which is of powder of SF6, becomes coarser as the thickness is increased, meanwhile, the surface are brighter. There are also many pits onto the coating surface caused floated slags from the molten pool, the pits on the $0.5 \mathrm{~mm}$ track is small and shallow while it is shaped like wrinkle as preplaced powder is $1 \mathrm{~mm}$ or $1.5 \mathrm{~mm}$. It also found that the particles is diminish while the diameter becomes larger. While Fig.4 d)-f) indicate the same tendency in surface roughness and brightness in laser cladding of Fe327 powder. As the preplaced powder thickness is $0.5 \mathrm{~mm}$, the irregular and interval grooves exist onto the track surface. However, the groove is turned to shallow pits when powder thickness is $1 \mathrm{~mm}$. Nevertheless, there is much furrow without distinct pit onto the surface when the powder is continued to add. Compared to SF6 cladding track, the particles onto the edge is totally less.

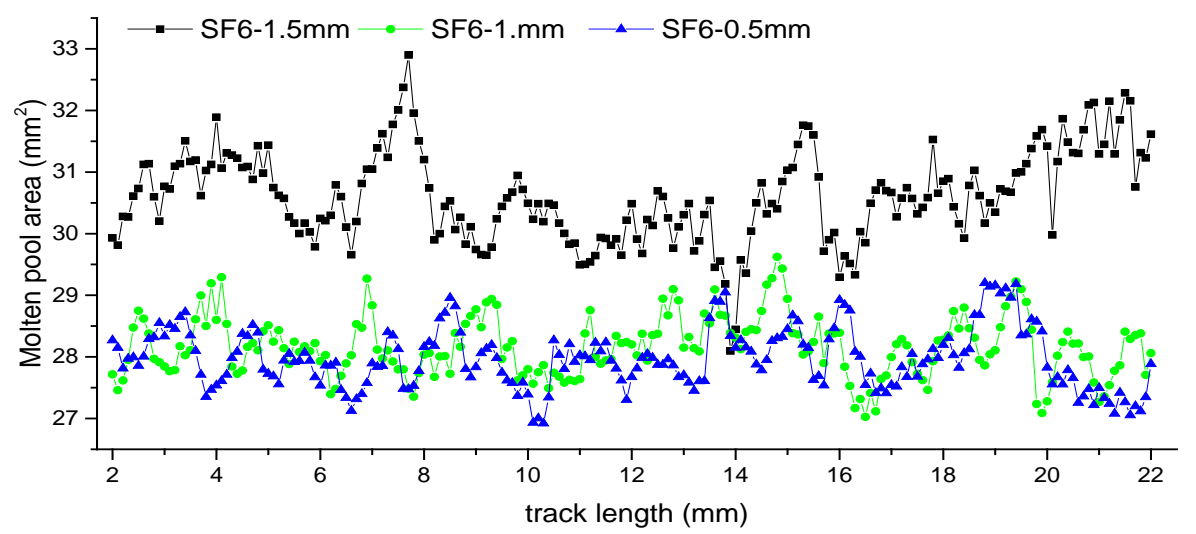

Fig.5 Area of cladding track with different preplaced SF6 powder thickness 


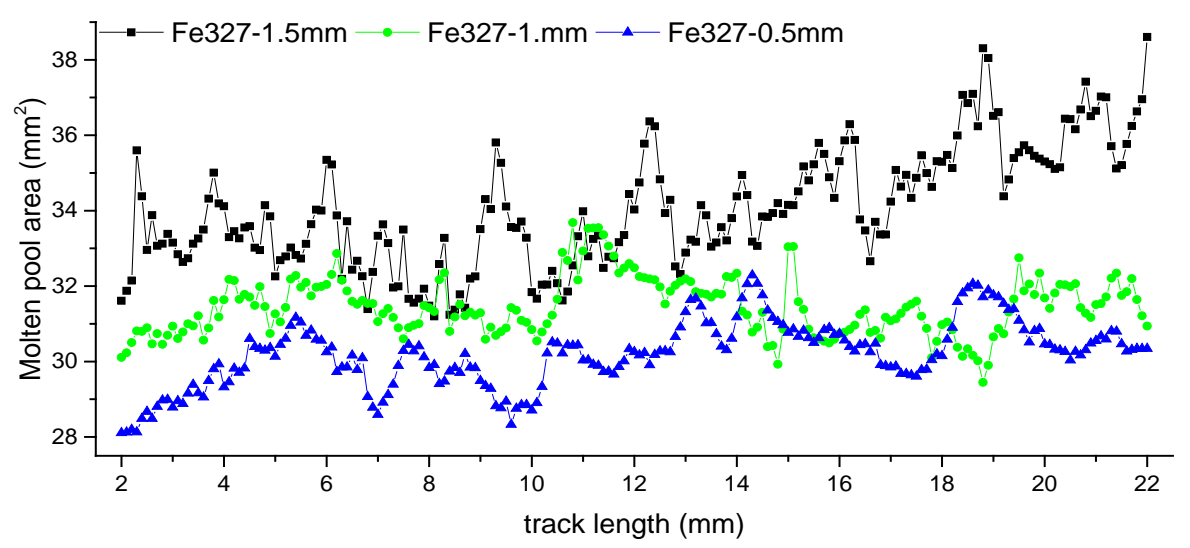

Fig.6 Area of cladding track with different preplaced Fe327 powder thickness

Fig. 5 and Fig. 6 shows the area of track captured and calculated by detection system, and the obvious rise happens when the thickness increase from $0.5 \mathrm{~mm}$ to $1.5 \mathrm{~mm}$. In process of Co-based powder SF6, the average molten pool area rises to $30.61 \mathrm{~mm}^{2}$ as the preplaced powder thickness is $1.5 \mathrm{~mm}$ while the average molten pool area is only $27.97 \mathrm{~mm}^{2}$ with preplaced powder thickness of $0.5 \mathrm{~mm}$, but also the average molten pool area of preplaced powder thickness of $0.5 \mathrm{~mm}$ and $1.0 \mathrm{~mm}$ is much closed, as shown in Fig.5. While in cladding of Fe327, the average molten area arise from $30.19 \mathrm{~mm}^{2}$ to $34.05 \mathrm{~mm}^{2}$. As a whole, the molten pool of Fe327 is larger than of the SF6, and the fluctuation of with thicker preplace powder is evidently larger, as shown in Fig.6.

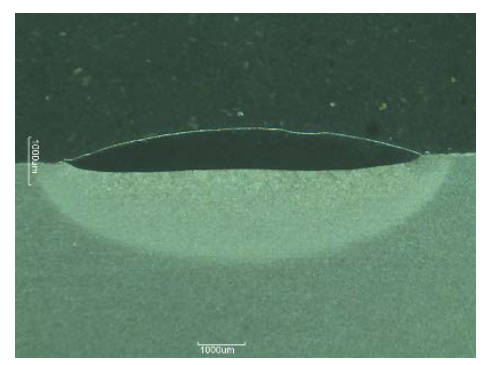

$0.5 \mathrm{~mm}$



$1.0 \mathrm{~mm}$

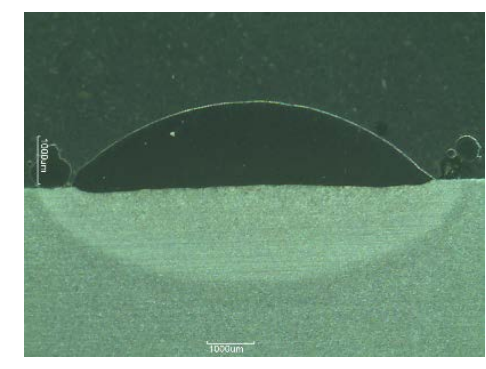

$1.5 \mathrm{~mm}$

Fig.7 Cross section of cladding bead with different powder thickness (SF6)



Fig.8 Geometrical parameters of cross section of cladding bead with different powers (SF6)

Fig 7 shows cladding bead geometries with different SF6 powder thickness, and the results of its width, height, depth of HAZ and dilution measured from optical observations are shown in Fig.8. From the view of Fig.8, the height increases with more powder in molten pool, while the width and HAZ show the same slightly increase as the thickness of powder is in progress. However, the dilution decreases as the powder is more. 


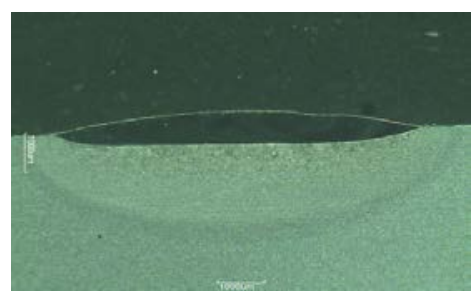

$0.5 \mathrm{~mm}$

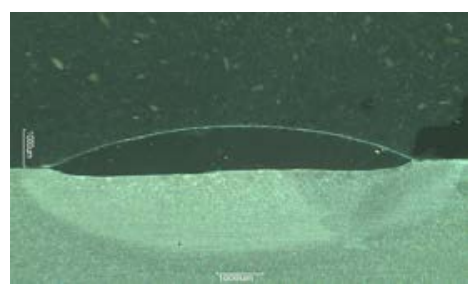

$1.0 \mathrm{~mm}$

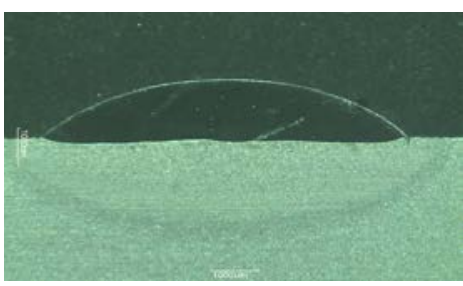

$1.5 \mathrm{~mm}$

Fig.9 Cross section of cladding bead with different powder thickness (Fe327)



Fig.10 Geometrical parameters of cross section of cladding bead with different powers (Fe327)

From Fig.9 and Fig.10, it can be observed that the change rule is nearly same as SF6 powder: the height and width of bead linearly increases as powder is added more, while the dilution and HAZ decreases with increase of powder thickness.

The molten pool is characterized by the shape of proximate tetragon due to the tetragonal laser spot, the area can be defined as product of the width of cross section by length of molten pool. However, the thick powder resulting in larger molten pool area (as shown in Fig.5 and Fig.6), which means the width or length is increased as a result. Since heat energy come from irradiation are absorbed by matrix and powder, the powder preplaced is thick need majority of energy to form molten pool, in turn, the minority of energy transfers to matrix, the dilution thus decreases.

\subsection{The multi-track cladding}

The coaxial powder feeding cladding were done on the material of ANSI 1045, the powder was Ni55. The cladding samples are listed in Fig.11, the whole cladding surface are basically flat without cracks.

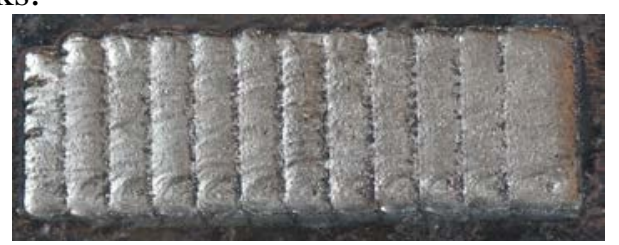

a. $3 \mathrm{~g} / \mathrm{min}$

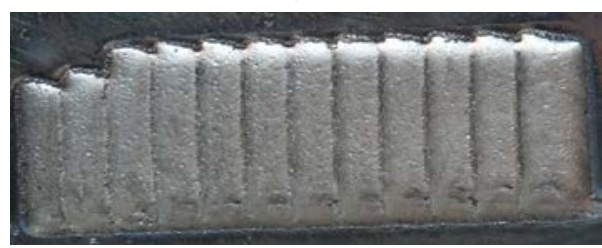

c. $9 \mathrm{~g} / \mathrm{min}$



b. $6 \mathrm{~g} / \mathrm{min}$

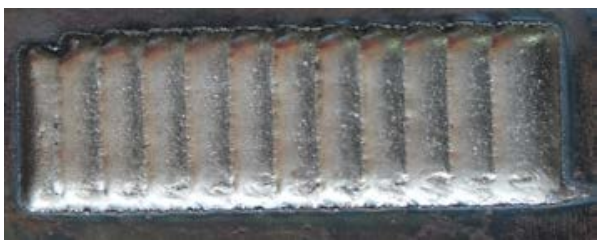

d. $12 \mathrm{~g} / \mathrm{min}$

Fig.11 Morphology of cladding layer with different powder flow rates

From the view of cladding layer's morphology, multi-track cladding layer becomes fine and smooth as the mass flow rate increases. Compared to $3 \mathrm{~g} / \mathrm{min}$ of mass flow rate, the surface waviness is small for each track and thus, the whole cladding surface becomes flat and smooth as mass flow rate is up to $6 \mathrm{~g} / \mathrm{min}$. No obvious slag is bonded with the upside surface, however, there are still some ripple on the beginning track surface. When the mass flow rate is to $9 \mathrm{~g} / \mathrm{min}$, the obvious uplift is appeared between two track overlapping areas and without slag is shown onto the 
up-surface. More obvious uplift, without slag and brightness are the characteristics of cladding surface as the flow rate is $12 \mathrm{~g} / \mathrm{min}$. On the average, the cladding layer becomes thicker with increased flow rate, which means that the laser power is enough for the selected feed capacity and therefore the good cladding behaviors.

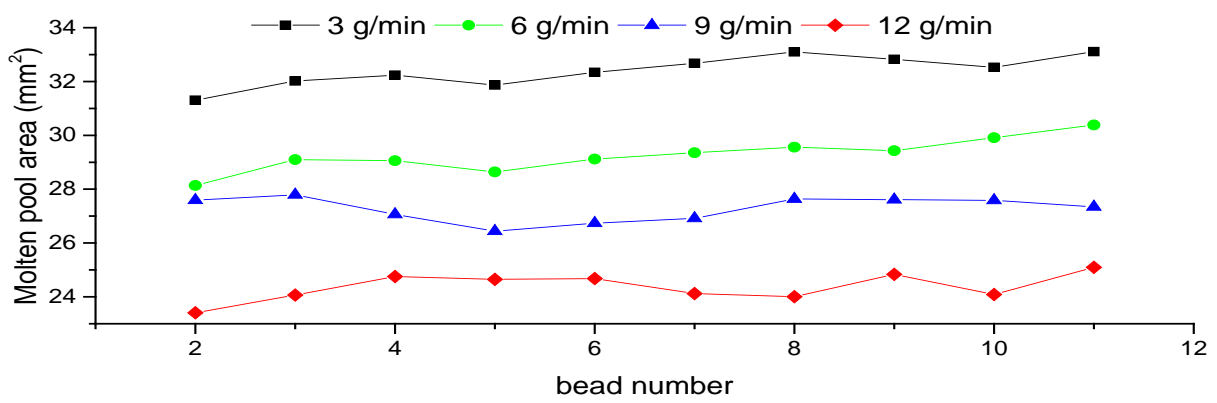

Fig.12 Relationship between flow rate ane molten pool area

Fig.12 reveals that the molten pool area decreases with the larger powder flow rate, and the increment of molten pool area is closed for every interval of $3 \mathrm{~g} / \mathrm{min}$. The larger powder flow speed cause the higher powder density in feeder head and thus shelter the irradiated laser and weaken the energy absorbed by substrate and deposited powder, as a result, the energy utilized to form the molten pool is decreased, so does the molten pool area. In Fig. it is also can be found that the area shows a trend to increase with continued cladding when flow rate is relatively small such as 3 or 6 $\mathrm{g} / \mathrm{min}$, whereas the molten pool area keeps in limited range as the flow rate is large. This result contributes to the accumulation affect in the process of cladding, when the feed powder is in small amount, the energy can melt the powder and substrate very quickly and save part of energy to heat the surrounding substrate material, ultimately, the more energy is used to melt the substrate and flowed powder and thus extend the molten pool area. While the energy absorbed by powder is in majority when the more powder is flowed to pool, which causes the energy transferred to substrate is limited thus, the molten pool area is kept in a range.

\section{Summary}

Laser cladding of SF6 and Fe327 with single track and multi-track Ni55 had been conducted on the ANSI 1045, the process of cladding was observed by CMOS camera vision system and area of molten pool was calculated so as to make sure the relationship between the quality of coating surface and powder quantity. And some conclusions have been drawn from the research as follow:

1) Molten pool had been captured by CMOS camera with appropriate image processing methods by LabVIEW program, and the area can be calculated from the image process system, which is beneficial to the quality of cladding control.

2) The different type of powder with different quantity had been conducted and discussed. Generally, the area increase as preplaced powder increased in powder of SF6 and Fe327, while it decreases as the Ni55 powder mass flow is much.

3) The works had been done verified that the CMOS vision detection is useful to ensure the quality of laser cladding surface.

\section{Acknowledgment}

The authors gratefully thank the financial support from National Natural science foundation of China (No 51275303) 


\section{References}

[1] J.T. Hofman, B. Pathiraj, J. van Dijk, D.F. de Lange, J. Meijer, Journal of Materials Processing Technology, 212 (2012) 2455-2462.

[2] M. Qian, L.C. Lim, Z.D. Chen, W.I. Chen, Journal of Materials Processing Technology, 63 (1997) 590-593.

[3] S. Zhou, X. Dai, X. Zeng, Applied Surface Science, 255 (2009) 8494-8500.

[4] G. Bi, A. Gasser, K. Wissenbach, A. Drenker, R. Poprawe, Optics and Lasers in Engineering, 44 (2006) 1348-1359.

[5] T. Terada, T. Yamada, A. Nishimura, in: SPIE LASE, International Society for Optics and Photonics, 2014, pp. 896310-896310-896316.

[6] I. Smurov, M. Doubenskaia, A. Zaitsev, Surface and Coatings Technology, 220 (2013) 112-121.

[7] M. Doubenskaia, M. Pavlov, S. Grigoriev, I. Smurov, Surface and Coatings Technology, 220 (2013) 244-247.

[8] M. Pavlov, D. Novichenko, M. Doubenskaia, Physics Procedia, 12 (2011) 674-682.

[9] F. Meriaudeau, F. Truchetet, Journal of Laser Applications, 8 (1996) 317-324.

[10] F. Meriaudeau, F. Truchetet, C. Dumont, E. Renier, P. Bolland, in: Signal Processing, 1996., 3rd International Conference on, 1996, pp. 1628-1631 vol.1622. 